\title{
La double fonction de l'eau dans La salle de bain de Jean-Philippe Toussaint
}

\section{David, Sylvain}

Université Concordia, sylvain.david@concordia.ca

\begin{abstract}
Resumen
La evocación recurrente de agua en La salle de bain (1985) de Jean-Philippe Toussaint refleja la angustia del narrador. Es también un comentario metatextual, enmarcando el régimen de cruce de texto. Por lo tanto, la metáfora acuática es de una doble función simbólica en la novela : tiene tanto una connotación negativa, ya que refleja un desgaste general (personal y colectivo), y positiva, en la medida en que ayuda a definir la escritura y la lectura, y así, de alguna manera, para aclarar el mismo medio por el que es posible romper con el estancamiento también postulado.
\end{abstract}

Palabras clave: Jean-Philippe Toussaint; La salle de bain; agua imaginaria; temporalidad; metatextualidad.

\section{Résumé}

L'évocation récurrente de l'eau dans La salle de bain (1985) de Jean-Philippe Toussaint reflète les angoisses du narrateur. Elle constitue également un commentaire métatextuel, cadrant les modalités de traversée du texte. La métaphore aquatique revêt dès lors une double fonction symbolique dans le roman : elle comporte à la fois une connotation négative, en ce qu'elle reflète une usure générale (tant personnelle que collective), et positive, dans la mesure où elle contribue à définir l'écriture et la lecture, et donc, d'une certaine manière, à préciser les moyens mêmes par lesquels il est possible de s'arracher à la stagnation par ailleurs postulée.

Mots-clés : Jean-Philippe Toussaint ; La salle de bain ; imaginaire de l'eau ; temporalité ; métatextualité.

\begin{abstract}
The recurrent evocation of water in La salle de bain (1985) by Jean-Philippe Toussaint reflects the anguish of the narrator. It also constitutes a metatextual comment, framing the interpretation of the text. Aquatic metaphor therefore holds a double symbolic function in the novel : it has both a negative connotation, in that it reflects a general erosion (both personal and collective), and positive, insofar as it helps to define writing and reading, and therefore, to somewhat clarify the very means by which it is possible to break away from the stagnation otherwise postulated.
\end{abstract}

Keywords : Jean-Philippe Toussaint ; La salle de bain ; imaginary water ; temporality ; metatextuality. 


\section{Introduction}

Le narrateur de La salle de bain de Jean-Philippe Toussaint, se retire, comme le suggère le titre du roman, dans cet espace isolé de son appartement pour fuir un monde extérieur qu'il estime pétri d' ' hostilité diffuse » (1985 : 117). Les efforts répétés de sa compagne Edmondsson et, surtout, le constat « qu'il n'était peut-être pas très sain, à vingt-sept ans, bientôt vingt-neuf, de vivre plus ou moins reclus dans une baignoire » (15), finissent toutefois par l'en faire ressortir après quelques jours. Il se heurte alors au chaos que représentent des artistes polonais recyclés en peintres en bâtiment, apparemment appelés à rénover sa cuisine, ce qui le pousse à s'enfuir à nouveau, cette fois pour un séjour en solitaire à Venise, où Edmondsson se résigne à « venir [1]e chercher » (68). Quelques querelles et autres non-événements plus tard, le narrateur est de retour à Paris, où il s'enferme une fois de plus dans sa salle de bain tout en ressentant, comme c'était déjà le cas au début du texte, un besoin de « compromettre la quiétude de [s]a vie abstraite » (123), laquelle se voit, là encore, mise à mal par l'angoisse que suscite son propre vieillissement. Le récit semble donc se terminer comme il commence, en une sorte d'éternel retour, si ce n'est d'inaction fondamentale.

Le point nodal du roman étant une salle de bain, le thème de l'eau paraît appelé à y tenir une grande importance. Or, si le narrateur décrit longuement son séjour dans la baignoire, celle-ci ne s'avère, en dépit du recours à un verbe aux connotations aquatiques, pas toujours pleine : « je coulais là des heures agréables, parfois habillé, tantôt nu » (11). Il précise en outre être «allongé » dans sa salle de bain vêtu d'« [u]n pantalon de toile beige, [d']une chemise bleue et [d']une cravate unie » (14), ce qui explique pourquoi il peut tour à tour y recevoir, sans gêne aucune, sa mère et un ami de la famille. Le tout fait contraste avec une scène où Edmondsson s'approprie momentanément la baignoire pour un usage plus conventionnel : «Des gouttes d'eau scintillaient sur son corps. Elle [...] donnait de petites claques sur la surface de l'eau. » (20) Le séjour du narrateur dans la salle de bain paraît dès lors, pour reprendre un jugement ironique de sa compagne, moins détrempé que « desséchant » (11).

L'eau revêt pourtant une importance symbolique primordiale dans le texte : elle apparaît dans l'humidité stagnante de la salle de bain, dans la pluie qui tombe constamment sur Paris, dans les canaux qui sillonnent Venise et dans la mer qui menace celle-ci au loin. La critique a maintes fois relevé l'omniprésence de ce thème chez Toussaint, sans pour autant en faire un objet d'étude principal. Sur un ton qui ne va pas sans rappeler celui de la narration, Olivier Bessard-Banquy constate par exemple que « [l]'eau est, si l'on peut dire, à la source des inspirations de l'auteur, elle baigne son imaginaire » (2003: 58), alors que Sylvie Loignon enchérit comme quoi « [1]a métaphore liquide imprègne l'œuvre » (2010 : §8). Toussaint lui-même, à l’occasion d’un entretien, reconnaît la forte présence de l'eau dans son imaginaire romanesque, sans pour autant daigner commenter davantage :

\footnotetext{
Il y a un certain nombre d'obsessions dans mes livres. Je peux les constater, mais je ne les théorise pas. L'eau en est une, capitale, extrêmement présente, mais il n'y a pas d'explication rationnelle à cela. C'est une obsession, ça me plaît, c'est mon désir, c'est mon plaisir. J'ai toujours éprouvé du plaisir à parler de l'eau, à la décrire, à faire des métaphores aquatiques, fluides, liquides, mouillées. C'est comme ça : j’en ai envie. Point (cité par Allemand, 2011 : 392).
}

Je tâcherai de montrer ici que l'eau revêt, dans le roman de Toussaint, une double fonction symbolique : elle comporte à la fois une connotation négative, en ce que son écoulement évoque le passage du temps et l'usure des choses, et positive, dans la mesure où sa fluidité se fait métaphore de l'écriture, de la création qui permet justement de transcender la finitude humaine.

\section{L’écoulement des instants}

\subsection{Une existence lézardée}

La salle de bain est avant tout le récit d'une décomposition individuelle. Le narrateur, obsédé par le passage du temps, contemple ainsi, de sa baignoire, la détérioration de la pièce qui lui rappelle sa propre condition mortelle : 
Le mur qui me faisait face, parsemé de grumeaux, présentait des craquelures ; des cratères ça et là trouaient la peinture terne. Une fissure semblait gagner du terrain. Pendant des heures, je guettais ses extrémités, essayant vainement de surprendre un progrès. Parfois, je tentais d'autres expériences. Je surveillais la surface de mon visage dans un miroir de poche et, parallèlement, les déplacements de l'aiguille de ma montre. Mais mon visage ne laissait rien paraître. Jamais (Toussaint, $1985: 12$ ).

Le brusque changement de perspective, qui passe de la cloison objet au miroir reflet du sujet, suggère une propension du narrateur à se concevoir en fonction de son environnement, un décalage qui se manifestera de façon récurrente dans la suite du récit. Comme la faille qui inquiète tant le personnage est vraisemblablement causée par cette «humidité omniprésente » (2010 : §29) qui, selon Alexandra Huguet, imbibe l’univers de Toussaint, l'eau comporte d'emblée, dans l’imaginaire du roman, une propriété dévastatrice.

La crainte obsessive de la mort se manifeste de plusieurs autres manières au fil de la diégèse. La scène du reflet et du chronomètre se répète, par exemple, presque à l'identique, dans un passage ultérieur : «Debout en face du miroir, je regardais mon visage avec attention. J'avais enlevé ma montre, qui reposait en face de moi sur la tablette du lavabo. La trotteuse tournait autour du cadran. Immobile. À chaque tour, une minute s'écoulait. » (Toussaint, 1985 : 25) De même, à l'occasion d'un souper en tête à tête au restaurant, le personnage offre à sa copine non pas, comme elle paraît s'y attendre, une bague, mais une « montre » (70). Enfin, il réagit fort négativement lorsque confronté à l' «affichett[e] qui fai[t] part d[u] décès » (79) d'un individu plus jeune que lui. Ces exemples, s'ils confirment les angoisses profondes du protagoniste, éloignent toutefois du thème de l'eau. Or, c'est dans l'aperçu offert du monde extérieur que celui-ci prend sa pleine importance.

\subsection{Une pluie diluvienne}

La salle de bain est également le récit d'une apparente décomposition collective. Celle-ci se voit signifiée, en un premier temps, par la pluie qui tombe incessamment sur Paris. De ce fait, chaque fois qu'il est question, dans la première partie du roman, du monde extérieur, c’est pour en rappeler la météo exécrable : «il pleuvait » (14); «il pleuvait à verse [...] : comme si toute la pluie allait tomber, toute » (36) ; «[1]es vitres étaient couvertes de pluie » (49). Les précipitations en viennent même, à l'occasion d'une parenthèse révélatrice de la narration, à se confondre avec la définition de la ville : «dehors, c'était toujours aussi parisien » (35). Les averses incessantes acquièrent ainsi, dans leur accumulation, une capacité cataclysmique qui fait écho à l'inquiétude fondamentale du narrateur. Se profilent alors des clichés de fin du monde, qui doivent autant à la parabole biblique du déluge qu'au mythe antique de l'Atlantide :

Il pleuvait. La rue était mouillée, les trottoirs étaient sombres. Des voitures se garaient. D’autres, en stationnement, étaient couvertes de pluie. Les gens traversaient la rue rapidement [...]. Un peu de vapeur commençait à recouvrir ma vitre. Derrière la fine pellicule de buée, j’observais les passants [...]. La pluie leur donnait des airs de conspirateurs [...]. J'approchai mon visage de la fenêtre et, les yeux collés contre le verre, j'eus l'impression que tous ces gens se trouvaient dans un aquarium. Peut-être avaient-ils peur? L’aquarium lentement se remplissait (30-31).

L'image du bocal, ou du réservoir, est, dans ce contexte, particulièrement révélatrice : elle évoque à la fois l'impuissance des citoyens, ainsi pris au piège, et l'isolement du narrateur, qui observe le tout à l'abri d'une cloison vitrée. Elle suggère en outre une opposition entre la décadence feutrée, mesurée, du protagoniste cloîtré dans son appartement ou sa salle de bain, et l'apocalypse brutale, directe, qu'il croît apercevoir à l'extérieur.

Cette submersion potentielle de Paris demeure toutefois, de l'aveu même du personnage, le fruit d'une imagination troublée :

je me disais que les gens ne redoutaient pas la pluie ; [...] nul n’avait vraiment peur qu'elle ne s'arrêtât plus jamais, écoulement continu faisant tout disparaître - abolissant tout. C'est moi qui, devant ma fenêtre, par une confusion que justifiait la crainte que m'avaient inspirée les divers mouvements qui se déroulaient devant mes yeux, pluie, 
déplacements des hommes et des voitures, avais eu soudain peur du mauvais temps, alors que c'était l'écoulement même du temps, une fois de plus, qui m’avait horrifié (31).

On en revient ainsi au constat initial d’une homologie, filée par le texte, entre intériorité et extériorité, laquelle demeure marquée par le potentiel dévastateur de l'élément liquide. Cette « union métaphorique du temps et des intempéries » (2003 : 58), comme l'a résumé Olivier Bessard-Banquy, offre toutefois une précision essentielle : si, dans l’immobilité de la salle de bain, l'écoulement du temps peut paraître momentanément contenu, la confrontation avec l'agitation du monde réel relance à l'inverse le mouvement mortifère. Le départ du narrateur de la France pour l’Italie offre d'ailleurs une variation révélatrice à cet égard.

\title{
1.3. Une ville engloutie
}

Alors que Paris était noyé sous la pluie, Venise peine à émerger des eaux qui la sillonnent. Une des rares descriptions faites de l'élément aquatique opère toutefois un rapprochement significatif entre ciel et voie navigable : « Le canal était sombre, noir comme les nuages. » (Toussaint, 1985 : 71) Les ténèbres imputées au cours d'eau prolongent ainsi les connotations négatives qui avaient été précédemment attribuées à l'averse. L’idée d'une action corrosive de l'élément liquide (ou, comme dans la salle de bain du narrateur, de l'humidité), est en outre soulignée par une plaisanterie géographique récurrente : «la peinture gondol[e]» (54); «le dallage de marbre [...] gondol[e] » (77); bref, tout se déglingue. De ce fait, chez Toussaint, comme le rappelle Sylvie Loignon, « c’est le mouvement même du temps et partant de la mélancolie qui se dit sous une métaphore liquide » (2010 : §8). La vision dystopique de Paris rejoint ainsi l'ambiance crépusculaire souvent associée à la cité des Doges.

Si chez lui, en France, le narrateur est terrifié par le passage du temps et l'usure des choses, à l'étranger, où, imagine-ton, il se sent moins concerné par les possibles conséquences de ses actes, il cherche au contraire à précipiter le mouvement. Une promenade faite avec sa compagne offre ainsi l'aperçu d'un curieux passe-temps :

\begin{abstract}
je marchais la tête baissée, en appuyant mes pas sur le trottoir pour enfoncer la ville dans l'eau. À chaque fois que je terminais la descente d'un escalier, je sautais discrètement à pieds joints sur le sol et, attendant Edmondsson au bas des marches, je l'invitais à faire de même. À raison d'un enfoncement de la ville de trente centimètres par siècle, expliquaisje, donc de trois millimètres par an, donc de zéro virgule zéro zéro quatre-vingt-deux millimètres par jour, donc de zéro virgule zéro zéro zéro zéro zéro zéro un millimètre par seconde, on pouvait raisonnablement, en appuyant bien fort nos pas sur le trottoir, escompter être pour quelque chose dans l'engloutissement de la ville (Toussaint, 1985 : 81).
\end{abstract}

Bien évidemment, le personnage ne peut que constater son impuissance à ce petit jeu, mais, peut-être que le fait de s'inscrire, pour une fois, du côté du chaos, au sein même de l'élan corrupteur, lui offre une ténue sensation de contrôle. Son obsession maniaque pour le temps demeure par ailleurs, comme le révèlent ses calculs détaillés, intacte.

\subsection{De l'eau morte à l'eau vive}

La submersion potentielle de Venise constitue ainsi une variation du déluge parisien, dans la mesure où l'une comme l'autre s'avèrent des projections de l'angoisse fondamentale du personnage. Un bref aperçu de son intériorité révèle, à cet égard, un lien direct entre l'élément marin, tel qu’observé à Venise, et sa mélancolie : « Lorsque, le matin, je me réveillais, je voyais la journée à venir comme une mer sombre derrière mes yeux fermés, une mer infinie, irrémissiblement figée. » (86) Similairement, en une métaphore étrange mais parlante, sa chambre lui paraît « un marais d’obscurité » (72), ce qui accentue d'autant plus l'idée d'une eau stagnante. La vision d'une mer mortifère, pétrifiée, qui engloutit irrémédiablement le sujet, se voit en outre réitérée lorsque, pressé par une fillette de lui raconter une histoire, le personnage choisit, de manière fort inappropriée, de lui narrer « le naufrage du Titanic » (104).

De ce fait, que l'intrigue se déroule à Paris ou à Venise, l'imaginaire de l'eau, dans La salle de bain, comporte une dimension éminemment menaçante, qui renvoie autant au passage du temps qu'à l'usure des êtres, des choses et des 
civilisations. De telles associations symboliques revêtent une importance fondamentale dans la mesure où, comme le rappelle Nicolas Xanthos, chez Toussaint, « [c]'est la métaphore liquide qui vient [...] permettre la représentation » (2009 : 83). Or, et c’est là l’une des grandes singularités de ce texte, si l'eau, au niveau de la diégèse, demeure ainsi un élément négatif, « une véritable “eau morte” » (2010 : §24) pour reprendre la formule d’Alexandra Huguet, à celui du récit, de la mise en écriture, elle s’avère à l’inverse un facteur positif, la source d'un dynamisme fécond.

\title{
2. La fluidité de l'écriture
}

\subsection{L'eau-mouvement}

Alors qu'il contemple la pluie de son appartement parisien, le narrateur se détourne momentanément de ses projections apocalyptiques pour s'intéresser à la chute de l'eau elle-même. Il en tire une réflexion à caractère philosophique, qui confirme et relance ses intuitions antérieures :

\begin{abstract}
Il y a deux manières de regarder tomber la pluie, chez soi, derrière une vitre. La première est de maintenir son regard fixé sur un point quelconque de l'espace et de voir la succession de pluie à l'endroit choisi ; cette manière, reposante pour l'esprit, ne donne aucune idée de la finalité du mouvement. La deuxième, qui exige de la vue davantage de souplesse, consiste à suivre des yeux la chute d'une seule goutte à la fois, depuis son intrusion dans le champ de la vision jusqu'à la dispersion de son eau sur le sol. Ainsi est-il possible de se représenter que le mouvement, aussi fulgurant soit-il en apparence, tend essentiellement vers l'immobilité, et qu'en conséquence, aussi lent peut-il parfois sembler, entraîne continûment les corps vers la mort, qui est immobilité. Olé (Toussaint, 1985 : 35-36).
\end{abstract}

Le ton de ce passage paraît d'emblée moins anxiogène, comme le suggèrent tant le protocole d'observation ainsi défini que le curieux « Olé » final, lequel vient dédramatiser le tout. Cette légèreté retrouvée du narrateur est probablement due au fait que, dans cette perspective davantage critique ou analytique, l'eau n'est plus la source du mouvement et de l'usure que celui-ci entraîne, mais simplement son médium, son incarnation momentanée qui, mue par une impulsion extérieure, permet d'en observer les effets. Se développe alors une «sémantique de l'eau qui tombe », selon la formule d'Olivier Bessard-Banquy, où « l'averse est métaphysique » (2003 : 58). L’aperçu offert de l'élément liquide dans le roman passe, par le fait même, du contenu au contenant, du fond à la forme.

Le constat posé par le narrateur lors de la contemplation des gouttes de pluie s’inscrit d’ailleurs dans le sillage d'autres observations du même acabit. Un voyage ferroviaire, par exemple, lui donne l'occasion de réfléchir au mouvement et à sa finalité :

J’avais passé la nuit dans un compartiment de train, seul, la lumière éteinte. Immobile. Sensible au mouvement, uniquement au mouvement, au mouvement extérieur, manifeste, qui me déplaçait malgré mon immobilité, mais aussi au mouvement intérieur de mon corps qui se détruisait, mouvement imperceptible auquel je commençais à vouer une attention exclusive, qu’à toutes forces je voulais fixer. Mais comment le saisir ? Où le constater (Toussaint, 1985 : 51) ?

De même, la dégustation d'un dessert glacé se fait le prétexte à une méditation complémentaire sur cette impossible « saisie » ou « fixation » du mouvement :

Je regardais la dame blanche fondre devant moi. Je regardais fondre imperceptiblement la vanille sous la nappe de chocolat brûlant. Je regardais la boule encore exactement ronde un instant plus tôt qui ruisselait lentement en filets réguliers blancs et bruns métissés. Je regardais le mouvement, immobile, les yeux fixés sur la soucoupe. Je ne bougeais pas. Les mains figées sur la table, j'essayais de toutes mes forces de garder l'immobilité, de la retenir, mais je sentais bien que, sur mon corps aussi, le mouvement s'écoulait (80). 
Si l'exemple du train ne comporte pas d'élément liquide, celui de la « dame blanche » oppose, de manière parlante, la congélation et la fonte, la fluidité et la consistance. Tout finit par couler ou s’écouler, suggère le texte, y compris la substance même du narrateur. De telles observations ne constitueraient toutefois qu'une réitération supplémentaire des obsessions du personnage si certains détails n’invitaient à y trouver une clé de lecture pour l'ensemble du texte.

\subsection{Le flux narratif}

À l'instar de ce qui est dit de la pluie par le narrateur, La salle de bain permet deux modalités distinctes, mais complémentaires, d’interprétation. Comme Toussaint l'a reconnu, dans le cadre d'un entretien, une « double lecture chronologique » du roman est possible :

La salle de bain peut [...] commencer soit au début de la première partie, soit au début de la deuxième partie. C'est pour cela qu'il y a trois points de suspension à la fin de la première partie, or je n’utilise pas beaucoup les points de suspension... Comme l'un des thèmes majeurs du livre est la relation entre l'immobilité et le mouvement, il se trouve que l'un de ces aspects chronologiques privilégie le mouvement et l'autre l'immobilité (cité par Ammouche-Kremers, $1994: 31)$.

Dans cette perspective, le texte peut être lu soit, dans sa séquence originelle, comme le récit d’une stagnation à Paris, d'une évasion momentanée à Venise et d'un retour à l'apathie parisienne, soit, en entamant la lecture au début de la deuxième partie (pour ensuite lire la troisième et revenir à la première), comme l'histoire d'une évasion à Venise, dont l'impulsion s'estompe progressivement pour laisser place à une immobilité parisienne, finale entropique qui se voit d’autant plus signifiée par des notations récursives, le séjour dans la salle de bain étant rapporté autant dans la première que dans la troisième partie.

De ce fait, comme il l'est préconisé pour les gouttes d'eau, le lecteur peut, d'une part, concentrer son attention sur le narrateur à l'occasion du passage réitéré de celui-ci dans un lieu fixe, en l'occurrence la salle de bain, et, d'autre part, suivre sa trajectoire de son origine à sa fin, ladite salle de bain constituant alors l'aboutissement du mouvement originel. La première de ces perspectives suggère, comme l'a indiqué Gil Delannoi, que « [1]'immobilité existentielle et cyclique est le fond de la mobilité apparente du récit », alors que la seconde, à l'inverse, présente « l'avantage de signaler les relations enchevêtrées du mobile et de l'immobile » (1985: 13). Si cette double lecture potentielle ne va pas sans rappeler certaines constructions narratives post-modernes, à l’interprétation éternellement ouverte, elle n’en a pas moins pour effet d'associer la trajectoire du narrateur à celle de l'eau qui s’écoule. Les deux entités ne sont de ce fait plus en opposition, mais se relancent, s’éclairent mutuellement.

\subsection{L’écriture-clepsydre}

Le rapprochement fait par le texte entre écoulement de l'averse et traversée diégétique invite ainsi, de manière plus abstraite, à creuser davantage la symbiose entre fond et forme, à penser conjointement les gouttes de pluie qui assaillent l'univers du roman et les fragments narratifs dont celui-ci est constitué. La salle de bain se présente en effet, à l'image des « Pensées de Pascal » (Toussaint, 1985 : 82) auxquelles la narration rend un hommage ironique, comme une série de courts paragraphes numérotés, d'un seul mot à une dizaine de lignes, dont l'enchaînement ne respecte pas toujours une stricte logique narrative. Cette construction paratactique invite, de prime abord, à focaliser sur ces blocs de texte en euxmêmes, à considérer ceux-ci comme autant d'arrêts sur l'image qui reflètent les préoccupations du narrateur pour l'immobilité, son impossible ambition de « saisir » (51) le temps ou « fixer » (51) le mouvement. Une telle approche se concentre, pour filer la métaphore pluviale, sur chaque goutte de manière statique, individuelle, sans forcément tenir compte de la « finalité » (36) de l'ensemble.

Or, le roman de Toussaint, malgré sa propension à « constater » (51) l'existence, n'est pas un recueil de maximes ou d’observations éparses : il est conçu pour être abordé dans la « succession » (36), c'est-à-dire, à l'instar de l'averse, dans la continuité. Chaque fragment, bien que pouvant être lu indépendamment, ne prend tout son sens, comme le rappellent notamment les multiples isotopies aquatiques qui sous-tendent le texte, que par rapport à l'ensemble qui le situe et le 
contextualise. De même, le narrateur, bien qu'affligé d'une apathie fondamentale, n'en suit pas moins une certaine trajectoire, tel que le suggèrent les deux modalités de lecture définies par l'auteur. La translation, effectuée par le roman, entre intériorité et extériorité prend ainsi, comme le signale Sylvie Loignon, tout son sens : « La pluie, [dans] La salle de bain, apparaît comme l'image même non seulement du temps mais de la pensée. » (2010 : §8) De ce fait, si la narration de Toussaint, représentative en cela du roman contemporain, ne repose pas, ou plus, sur une intrigue classique, elle comporte tout de même une impulsion intrinsèque, un mouvement d'ensemble.

\subsection{Une fuite perpétuelle}

L'opposition, qui se manifeste dans la forme même de La salle de bain, entre flux et tarissement, écoulement et contention, ne va pas sans rappeler la description offerte, par le texte, d’un canal vénitien : « L’eau, un instant figée, emmurait le perron d'une église, puis fondait en cascade, abandonnait les marches une à une. » (Toussaint, 1985 : 71 ) Cette image, métaphore apparente de la dynamique narrative du roman, illustre de manière emblématique le constat de Sophie Bertho comme quoi, chez Toussaint, « le découpage du récit en séquences numérotées [...] montre de façon visible l'immobilisation du temps sous l'apparente fluidité du récit » (1994 : 19). Elle suggère en outre que la contention d'un flot ne peut être que momentanée ; qu’une telle contraction a pour effet, dans le cas de l'élément liquide, de mener à un surcroît de pression, lequel se traduit inéluctablement par un débit subséquent accru. En d'autres mots, l'eau temporairement contrainte finit par couler avec d'autant plus de vigueur. Conséquemment, selon la logique de la métaphore aquatique, l'entrave faite, par le biais du fragment, au flux narratif en vient, paradoxalement, à mieux relancer celui-ci.

Cette tension, fondamentale au niveau formel, trouve une série de résonances au niveau thématique. D’une part, les actions du narrateur, obsédé par le passage du temps qu'il associe à l'écoulement de l'eau, visent à tenter de maîtriser, ou contraindre, le mouvement aquatique. Il privilégie ainsi l'espace symbolique de la salle de bain où l'eau est, par le biais d'un système élaboré de tuyauterie, asservie, contenue. De même, sa destination de prédilection est Venise, où, là encore, à la suite d'une complexe intervention humaine, les flots sont endigués, canalisés. La volonté du personnage de dominer les éléments s'avère cependant futile, dans la mesure où le propre d'un fluide, comme d'un instant, est de constamment fuir, de demeurer insaisissable. En conséquence, dans le roman de Toussaint, tout finit, inéluctablement, par faire flux. Les choix de verbes, typiques des jeux de mots de l'auteur, confèrent à cet égard un mouvement implicite, mais significatif, à l’ensemble : le narrateur « coul[e] des heures agréables » dans sa salle de bain (Toussaint, 1985:11); « [l]es après-midi s’écoul[ent] paisiblement » (60) ; un transistor « écoul[e] du rock and roll » (55) ; etc. Loin de comporter une connotation péjorative, cet ultime aspect des choses vient conférer une ouverture au roman, une « ligne de fuite » si l’on veut, pour filer à son terme la métaphore liquide.

\section{L'eau réflexive}

\subsection{Un symbole équivoque}

La représentation de l'eau dans La salle de bain revêt ainsi, comme il l'a été postulé en introduction, des qualités simultanément positives et négatives, dont la pondération globale fluctue selon la perspective retenue. Si, au niveau de la diégèse, le thème de l'écoulement est lié à celui du passage du temps et comporte dès lors une dimension mortifère, à celui du récit, de la forme romanesque, il renvoie au flux de l'écriture, à la limpidité du style. Ce caractère équivoque de l'élément aqueux, bien qu'exacerbé par l'usage constant que fait Toussaint de « métaphores aquatiques, fluides, liquides, mouillées » (cité par Allemand, 2011 : 392), ne déroge pas à la symbolique qui lui est traditionnellement associée. Jean Chevalier et Alain Gheerbrant considèrent en effet que «[l]es eaux, masse indifférenciée, représentent l’infinité des possibles, elles contiennent tout le virtuel, l’informel, le germe des germes, toutes les promesses de développement, mais aussi toutes les menaces de résorption » (1982 : 374). Une telle ambivalence, qui appelle à un équilibre précaire, sans cesse à renégocier, ne va pas sans rappeler celle du texte lui-même, dont la narration cumule les paradoxes et les dichotomies sans jamais véritablement trancher. 


\title{
3.2. Un monde en devenir
}

La suite du parcours romanesque de Toussaint offre une variation intéressante sur ce thème, en ce que les personnages ne se contentent pas, ou plus, de contempler l'eau : ils y plongent. S'il pleut encore abondamment dans des textes comme L'appareil photo ou La vérité sur Marie, et si l'action se déroule encore à proximité de la mer dans La réticence ou Fuir, il est révélateur de constater que, à compter de La télévision, de nombreuses scènes sont consacrées à la pratique enthousiaste de la natation. Un passage de Faire l'amour paraît emblématique de cette tendance :

\begin{abstract}
Je nageais [...], respirant doucement en laissant mes pensées se fondre dans l'harmonie de l'univers. J'avais fini par me déprendre de moi, mes pensées procédaient de l'eau qui m’entourait, elles en étaient l'émanation, elles en avaient l'évidence et la fluidité, elles s'écoulaient au gré du temps qui passe et coulaient sans objet dans l'ivresse de leur simple écoulement, la grandeur de leur cours, comme des pulsations sanguines inconscientes, rythmées, douces et régulières, et je pensais, mais c'est déjà trop dire, non, je ne pensais pas, je faisais maintenant corps avec l'infini des pensées, j’étais moi-même le mouvement de la pensée, j’étais le cours du temps (Toussaint, 2002 : 52).
\end{abstract}

De ce fait, l'élément liquide, source de menace lorsqu'il était contemplé de l'extérieur, comme c'est le cas dans La salle de bain, se mue en image même de l'harmonie, si ce n'est de la transcendance, quand il est appréhendé de l'intérieur. En une évolution concomitante, l'écriture de Toussaint délaisse le minimalisme et le fragment, voués, si l'on en croit certaines remarques métatextuelles, à contenir le mouvement, pour investir pleinement la fluidité, ainsi qu'en témoignent notamment les longues phrases d'inspiration proustienne qui sous-tendent les romans du « Cycle de Marie ».

Une telle «disposition océanique » (Toussaint, 2013 : 36) constitue peut-être la solution, apparemment hors de portée pour le narrateur de La salle de bain, au dilemme posé par l'écoulement conjoint de l'eau et du temps. Si l'élément liquide, comme le monde extérieur, paraît inquiétant lorsqu'observé d'une position de retrait, une manière de conjurer ses effets potentiellement délétères serait de s'y immerger pleinement, d'en suivre le courant, de se fondre dans son flux. L'héroïne évanescente de Nue connaît, à cet égard, une épiphanie significative : « Marie n’avait encore jamais envisagé de travailler consciemment sur ce qui échappe. Non, elle voulait toujours tout contrôler, sans voir que ce qui lui échappait était peutêtre ce qu'il y avait de plus vivant dans son travail. Car la perfection ennuie, alors que l'imprévu vivifie. » (25) Il en va possiblement de même pour la représentation de l'eau, dans l'œuvre de Toussaint. Ce serait dans les fuites, terme cher à l'auteur, auxquelles invitent son caractère informe et sa fluidité que résiderait la force de l'élément liquide, l'impulsion qu'il communique à qui a le courage de s'y abandonner, de se laisser emporter par la vague.

\section{Références bibliographiques}

Allemand, Roger-Michel (2011). «Jean-Philippe Toussaint : la forme et la mélancolie [entretien] », dans @nalyses, vol. 6, nº 1, p. 384-403. <https://uottawa.scholarsportal.info/ojs/index.php/revue-analyses/article/view/773/675> [Consulté le 14 février 2016].

Ammouche-Kremers, Michèle (1994). «Entretien avec Jean-Philippe Toussaint », dans Ammouche-Kremers, Michèle et Hillenaar, Henk. Jeunes auteurs de Minuit. Amsterdam : Rodopi, p. 27-36.

Bertho, Sophie (1994). «Jean-Philippe Toussaint et la métaphysique », dans Ammouche-Kremers, Michèle et Hillenaar, Henk. Jeunes auteurs de Minuit. Amsterdam : Rodopi, p. 15-26.

Bessard-BAnquy, Olivier (2003). Le roman ludique. Jean Échenoz, Jean-Philippe Toussaint, Éric Chevillard. Lille: Presses universitaires du Septentrion.

Chevalier, Jean et GheErbrant, Alain (1982). Dictionnaire des symboles. Paris : Robert Laffont / Jupiter.

DelanNoI, Gil (1985). «Cruel Zénon », dans Critique, n 463. Repris dans Demoulin, Laurent (2005). La salle de bain. Revue de presse. Paris : Minuit, p. 12-13.

Huguet, Alexandra (2010). «"Décourager la réalité”?», dans Recherches \& Travaux, $\mathrm{n}^{\circ} 77$. <http://recherchestravaux.revues.org/430> [Consulté le 27 février 2016]. 
Loignon, Sylvie (2010). «Comment finir? La mélancolie de Jean-Philippe Toussaint », dans Textyles, $\mathrm{n}^{\circ} 38$.

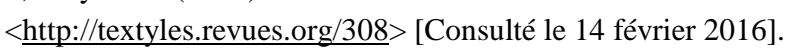

ToussainT, Jean-Philippe (2013). Nue. Paris, Minuit.

ToussAint, Jean-Philippe (2002). Faire l'amour. Paris : Minuit.

Toussaint, Jean-Philippe (1985). La salle de bain. Paris : Minuit.

XANTHOs, Nicolas (2009). « Le souci de l'effacement », dans Études françaises, vol. 45, nº 1, p. 67-87. 